The Overrepresentation of Aboriginal Women in Prisons:

A Cycle of Victimization, Discrimination and Incarceration

Alysa Holmes 
$\underline{\text { Introduction }}$

Throughout recent history, there has been an increased awareness of the overrepresentation of Aboriginal peoples in prison. While this issue certainly impacts Aboriginal peoples as a whole, this overrepresentation is most noticeable amongst the population of female offenders. Despite making up a relatively small portion of the Canadian population, research suggests that $\tilde{\text { B } 5.5 \% ~ o f ~ i n c a r c e r a t e d ~ w o m e n ~[a r e] ~ o f ~ A b o r i g i n a l ~ a n c e s t r y o ̀ ~(O f f i c e ~ o f ~ t h e ~}$ Correctional Investigator, 2015). Although the issue of overrepresentation has come to the forefront of research into specific prison populations, the increased attention to this topic has perhaps been influenced by the fact that this overrepresentation has actually become worse over time. For example, ñin the ten year period between March 2005 and March 2015é the number of Aboriginal women inmates doubledò(Office of the Correctional Investigator, 2015). As this overrepresentation is so dramatic in comparison to other minority groups, and the problem has only gotten worse over time, it gives rise to the question of why this specific population is so frequently in contact with the law. Through their frequent victimization, both historically and in a contemporary context, their mistreatment by the police and the court system, as well as their experiences during incarceration, Aboriginal women are often placed at a severe disadvantage both in the justice system and in society in general. This disadvantage sets in motion a cycle of victimization and offending behaviors, ultimately leading to their severe overrepresentation in the prison system. 
$\underline{\text { Victimization and Offending Behaviours }}$

\section{Contemporary Experiences of Violence}

One of the primary reasons that Aboriginal women are overrepresented as offenders is, perhaps counterintuitively, their overrepresentation as victims of violence and discrimination. As Fillmore and Dell argue, ñwomen who come into contact with the criminal justice system are commonly victims ofé abuse, violence and neglectò(as cited in Dell \& Kilty, 2013). This notion is especially relevant to the offending behaviors of Aboriginal women due to the long history of abuse against Aboriginal peoples, as well as the marginalized position that they occupy as a result of the intersection of their race and gender. As a population, Aboriginal women experience abuse and violence at incredibly high rates, with Aboriginal women three times more likely to die as a result of violence than non-Aboriginal women (Dylan, Regehr, \& Alaggia, 2008). While this number is significant, these high rates of victimization are even more blatant when considering Aboriginal women who are also offenders. According to Restoule (2009), ñ90 percent of Aboriginal women offenders report physical abuseé and 61 percenté report sexual abuseò (263). This clearly demonstrates that a large overlap exists between victims and offenders, and should be seen as evidence that a strong relationship does exist between victimization and offending behaviors.

However, this raises the question of why the experience of being victimized so often leads to offending. Research suggests that it is not always the actual experience of abuse itself that causes these individuals to offend, but rather that the offending behaviors in question are often a by-product of attempts to survive the experience of violence. For example, many victims become involved in some form of áleviantôbehavior, such as substance abuse, as a method of 
coping (Dell \& Kilty, 2013; Jackson, 1999). The types of crimes associated with these behaviors are perhaps more common amongst female Aboriginal victims of violence and abuse, as their already marginalized position in society limits the legitimate coping and survival strategies that are available to them. Although it is perhaps possible to attribute the high rates of victimization experienced by these offenders to their gender, these numbers are drastically higher than those pertaining to female offenders that are non-Aboriginal. For example, according to the Canadian Task Force on Federally Sentenced Women (1990), 68 percent of non-Aboriginal female offenders report a history of physical abuse, and only 53 percent report experiencing some form of sexual abuse (as cited in Jackson, 1999). Despite the fact that these numbers are still high, and should be seen as problematic, the gap between the statistics for Aboriginal female offenders and those of non-Aboriginal descent clearly suggests that race plays a significant role in increasing the likelihood of being a victim of violence and abuse. Overall, this demonstrates that there is a strong relationship between victimization and offending behaviors and, subsequently, that the high rates of violence experienced by Aboriginal women should be seen as directly linked to their high rates of incarceration. However, it also raises the question of why being Aboriginal so drastically increases the likelihood of experiencing violence.

\section{The Impact of Historical Trauma}

While research has focused on the impacts of contemporary abuse and violence on Aboriginal women as individuals, it has perhaps neglected an examination of their historical victimization through colonizing efforts such as residential schooling. While overrepresentation in prisons is most noticeable in regards to Aboriginal women, historical victimization has had a significant impact on the offending behaviors of many Aboriginal people, regardless of their 
gender. As such, before turning to the gendered aspects of colonization, it is vital to examine how these colonizing efforts have victimized Aboriginal peoples as a whole. According to Martel and Brassard (2008), the overrepresentation of Aboriginal peoples should be considered linked to the loss of identity that these peoples have suffered as a result of colonization. While this loss of identity should be seen as a form of victimization, it is important to note that colonization works to victimize Aboriginal peoples in many, perhaps more tangible, ways.

At a general level, research suggests that as a result of being subjected to the dispossession of ñland, [culture] and governmental authorityé Aboriginal peoples in Canada have a [long] history of social and economic disadvantageò (Martel \& Brassard, 2008, 343). This disadvantage should be considered strongly linked to the offending behaviors of Aboriginal peoples as, by pushing them out of work and confiscating their land, it places these peoples into a marginalized position in society, removing legitimate opportunities, and ultimately raising the likelihood that they will come into contact with the justice system. These forces have had an especially strong impact on the position of Aboriginal women in society, as ñthe percentage of Aboriginal women living in poverty is more than double the percentage of non-Aboriginal womenò (Aboriginal Affairs and Northern Development, 2012, 59). Although the extreme state of economic deprivation experienced by these women does explain why the issue of overrepresentation is even further exacerbated for Aboriginal women than it is for Aboriginal men, it is not immediately obvious why colonization has arguably had further negative impacts on Aboriginal women. As research suggests, Aboriginal women face áompound discrimination,ô as they are not only viewed as inferior due to their race but also as a result of their gender (Restoule, 2009). This perhaps explains why Aboriginal women, rather than Aboriginal men, are 
so often victimized, despite both experiencing the process of colonization. This suggests that while colonization has damaging effects on Aboriginal populations as a whole, it is also in many ways a gendered experience, with men and women being treated very differently under the rule of the colonizing force. According to Restoule (2009), the process of colonization has worked to create stereotypes of Aboriginal women as having little value or worth, not only to their own peoples but to society at large. The creation and perpetuation of these stereotypes have ultimately worked to paint Aboriginal women as acceptable targets for violence (273).

Although it is evident that these general impacts of colonization have certainly functioned to victimize Aboriginal women, specific colonizing efforts, such as residential schools, have had even more noticeable effects. While research does suggest that the abuses that many Aboriginal peoples suffered in residential schools have had severe impacts on these individualsômental and physical health (Bombay, Matheson, \& Anisman, 2014), these effects should not be seen as isolated to the direct survivors of these institutions. Through the concept of historical trauma, this abuse has worked to victimize further generations of Aboriginal peoples. According to Bombay et al. (2014), ñthe more generations that attended [residential schools], the poorer the psychological wellbeing of the next generationò(331). Additionally, this historical trauma also plays a role in impacting an individualô ability to cope with the stresses of contemporary forms of discrimination and violence (330). As such, colonization continues to victimize Aboriginal women in many ways, by placing them in a marginalized position in society, in terms of both financial and social status, and subsequently, through the experience of historical trauma, making them less able to cope with the violence that they experience as a result of this marginalization. As it has previously been established that victimization plays a strong role in the offending 
behaviors of women, the colonial victimization of Aboriginal women should be considered a significant factor in their overrepresentation in prisons.

\section{Policing Practices}

Despite the key role played by victimization in explaining the offending behaviors of Aboriginal women, it should not be considered the only cause of their high levels of involvement with the justice system. In fact, systemic disadvantages in dealing with the justice system play a large role in contributing to this overrepresentation. This is most evident in an examination of policing practices that unfairly target both Aboriginal women specifically, and Aboriginal peoples as a whole. The primary way that this disadvantage manifests itself is through overpolicing, wherein Aboriginal peoples are targeted by the police based on assumptions rooted in stereotypes (Manitoba, Public Inquiry, 1991). As policing efforts are often focused on q́problem areasôcharacterized by poverty, and Aboriginal peoples are disproportionately members of a lower socioeconomic class, there is subsequently an increased level of policing of Aboriginal peoples. While the experience of over-policing does apply to Aboriginal peoples as a whole, the emphasis on poorer areas again targets Aboriginal women, due to the extremely high levels of poverty that they face. As Pasmeny (1992) suggests, r̃onstant and excessive patrollingé results in the categorization and stigmatization of [Aboriginal peoples $\hat{q}$ behavior so that police officers tend to stop a higher proportioné for minor offenses, simply because they believe that Aboriginal people tend to commit more crimesò (406). This notion is clarified by Quigley (1990), who states that this type of policing leads to ña self-fulfilling prophecy, [where] they tend to police areas frequented by the groups that they believe are involved in criminality (as cited in Pasmeny, 1992, 406). As this demonstrates, over-policing tends to single out the behaviors of 
Aboriginal peoples, simply as a result of widely held stereotypes. This ultimately feeds back into itself, by reinforcing the notion of Aboriginal peoples as criminals.

These stereotypes are further exacerbated when they are applied to Aboriginal women, in many ways serving to criminalize these women even when they are the victims of an offense. Primarily, the victimization of these women is frequently disregarded or mishandled by the police, as evidenced by the underwhelming response given to missing Aboriginal women (McGillivray, as cited in Balfour, 2013; Razack, 2000). Although this lack of response does not directly criminalize these women, it creates an implication that some blame rests on the victim. By ignoring the victimization of these women, the police are reinforcing the notion that Aboriginal women are acceptable targets of violence. This only serves to further victimize these women, which, as previously discussed, can have a large impact on their future offending behaviors. Although this passive form of criminalization should be considered extremely problematic, perhaps more damaging is how these women are actively punished for their victimization. Research suggests that p̃police [are] more likely to counter-charge low income and racialized women when responding to domestic violence callsò(Comack et al; Pollack et al. as cited in Balfour, 2013, 94). This blatant discrimination means that Aboriginal women are often charged not only when they are innocent of a crime, but also when they themselves are the victims of the crime in question. This contributes greatly to their subsequent overrepresentation, as it creates multiple pathways to prison for these women. Regardless of whether you are a victim or an offender, the end result may be the same. Once again, this active form of discrimination is rooted in stereotypes of ñAboriginal women as [a] licentious and dehumanized squaw,ò(Razack, 2000, 193) ultimately laying blame on them for their own victimization. As 
such, through these discriminatory practices that disproportionately target Aboriginal women and paint them as offenders, current policing practices play a significant role in contributing to severe overrepresentation of Aboriginal women in Canadian prisons.

\section{Issues at Sentencing}

However, this systemic discrimination should not be considered limited to policing and is, in fact, evident throughout the court system as well. Although the disadvantage that Aboriginal women face at this stage is less blatant than that found at the level of policing, it is especially salient in explaining the overrepresentation of Aboriginal women in the Canadian prisons. As a population, when dealing with the court system, Aboriginal peoples ñare more likely to be denied bailé not have legal representationé [and are] more than twice as likely to be incarcerated than non-Aboriginalsò(Restoule, 2009, 259). As this demonstrates, Aboriginal peoples face a severe disadvantage in judicial proceedings, a factor which should be seen as directly linked to their overrepresentation. Dell suggests that Aboriginal women, in particular, face stigmatization and misrepresentation at sentencing (as cited Dell \& Kilty, 2013). Although research supports the notion that Aboriginal women are treated unfairly in the court system, ultimately increasing the likelihood that they will be incarcerated, the implementation of special sentencing practices has perhaps created the perception that this disadvantage does not exist.

While it may be argued that special sentencing practices, such as those arising from $\mathrm{R} \mathrm{v}$ Gladue, have worked to remedy this disadvantage, or even provide an advantage to Aboriginal peoples, these practices should be considered ineffective, especially in regard to the sentencing of Aboriginal women. This is most evident in judgesôreluctance to apply these principles. For example, it has been suggested that this ñspecial considerationé for Aboriginal offenders, [along 
with]é non-carceral sentencing alternatives [,] are pursued most often on behalf of [Aboriginal] mené and seldom on behalf of Aboriginal womenò(Balfour, 2013, 86). This is severely damaging to the equality of Aboriginal women in court, as they are less likely to actually have these background factors considered. Thus, these principles lend an appearance of equality within the system, suggesting that no further reforms need to be made to the sentencing process. Ultimately, these principles cannot be considered effective if they are not actually being applied. However, this is not to suggest that the implementation of special consideration eliminates the disadvantage that these women face. Williams (2007) suggests that when Gladue principles are applied, it often results in the overemphasis of historical and ancestral issues, reinforcing the notion that the criminality of Aboriginal women is inevitable, and ultimately shifting the focus away from more immediate criminogenic factors. This improper application of these principles functions to further disadvantage Aboriginal women at sentencing, by disregarding the individual circumstances of the case at bar. As such, it is important to strike a balance between an understanding of the contextual factors contributing to the disproportionate amount of contact that Aboriginal peoples have with the criminal justice system and the recognition that the overemphasis of these factors can lead to generalizations that further disadvantage Aboriginal women.

Beyond special consideration of background factors, Aboriginal women should also be seen as disadvantaged at sentencing by preconceived notions about the justice system held by the court itself. This is illustrated by the fact that ñsentencing judges are often under the impression that Aboriginal people will have access to culturally appropriateé programs, therapies, and resources for healing if they are sentenced to federal timeò (Milward, Parkes, 2011, 105). This 
should be considered incredibly problematic, as it ultimately punishes Aboriginal offenders beyond what they deserve by subjecting them to longer sentences, harsher institutions, and the stigma associated with being a federal offender. Although the fact that overrepresentation is actually worse at the provincial level, with ñAboriginal females account[ing] for $38 \%$ of female admissions to provincial [and] territorial sentenced custody,ò(Reitano, 2016, 5) gives rise to the argument that the misuse of federal sentences is rare, and has a negligible impact, this criticism should be considered extremely flawed. In many cases, the offenses these women have committed are relatively low-level crimes of survival and are thus unlikely to result in a federal sentence, regardless of judicial discretion. As such, while overrepresentation in provincial institutions demonstrates that judicial reliance on federal sentences as a pathway to treatment is not the sole cause of the overrepresentation of Aboriginal women in prisons, it should still be considered a significant contributing factor.

Additionally, while the tendency to default to a federal sentence certainly impacts both male and female offenders, the problems that it creates are exacerbated when considering female Aboriginal inmates. As many programs offered to women are only available at medium and minimum security levels, and Aboriginal women are particularly heavily overrepresented at the maximum security level, the majority of these women are excluded from these programs entirely (Restoule, 2009). As such, the use of harsher sentences as a way to provide access to treatment and programming should be considered ineffective and paternalistic. As well, this reliance on prison programming to meet the treatment needs of female Aboriginal offenders simply provides an excuse for the lack of programming available in the community. Overall, Aboriginal women are severely disadvantaged throughout the sentencing process in many different ways. Despite 
the introduction of special consideration for Aboriginal offenders through the principles found in Gladue, little has been done to effectively address this discrimination, resulting in the courts continuing to contribute to the high rates of incarceration experienced by Aboriginal women.

\section{Carceral Experiences}

\section{Classification of Offenders}

As previously discussed, Aboriginal women face discrimination and disadvantages at all stages of the justice system, thus contributing significantly to their overrepresentation in prisons. However, it is important to note that these disadvantages are also present in Aboriginal womenô experiences of incarceration, resulting in longer terms and increased rates of recidivism, and thereby contributing to their overrepresentation. One of the primary ways that Aboriginal women are disadvantaged while incarcerated is through over-classification based on preconceptions about these women, and the misuse of risk assessment tools. For example, current assessment tools consider ñ low level of education or employment training, past experiences of violenceé [and any] addiction[s] ò(McGill, 2008, 99) as indicative of an offender with a high level of needs, which subsequently corresponds to a high level of risk. As many of these factors are incredibly prevalent amongst female Aboriginal offenders, the use of this tool unfairly singles them out based on background factors that are often out of their control. While it may be argued that this test is ultimately an objective tool, Restoule (2009) suggests that the offender intake assessment (OIA) was developed based on the standard of a male, likely Caucasian, offender, and is thus inappropriate for use on women and individuals from different cultural backgrounds, resulting in these offenders being classified as posing a much higher risk than they actually do. The assignment of this ớigh riskôlabel to these offenders subsequently exacerbates 
the discrimination that these women face while in prison, as it works to reinforce the stereotype of Aboriginal women as unpredictable and violent offenders.

This perhaps justifies the mistreatment that this population faces while in prison. According to McGill (2008), ñfederally sentenced Aboriginal women are likely to experience harsher treatmenté [by Correctional Service Canada] staff than their non-Aboriginal counterpartsò (100). This harsh treatment further victimizes many of these women, which, as previously discussed, feeds back into their offending behaviors. While this harsh treatment on an individual level is certainly problematic, this discrimination occurs at a systemic level as well. For example, these women are frequently placed in administrative segregation for long periods of time and are less likely to receive either day parole or full parole than non-Aboriginal female offenders. Additionally, Aboriginal women convicted of violent offenses often end up serving much longer sentences than non-Aboriginal women convicted of similar offenses (Canadian Human Rights Commission, 2003; National Parole Board, 2000 as cited in Restoule, 2009). As this demonstrates, Aboriginal women are generally kept in prison longer than non-Aboriginal women, thus contributing to their overrepresentation in the population of incarcerated women. As well, because these women are often subject to harsher treatment while in prison, they are in many ways victimized, ultimately increasing the likelihood that they will re-offend. However, perhaps most problematic is the negative impact that over-classification can have on their access to treatment and programming. As previously established, programming is often not available to ónigh-riskôoffenders, thus this misclassification creates a barrier between Aboriginal women and the treatment that they require. As such, these women are often released without having received the programming that they need to treat the issues that led them to offend in the first place, and 
that would ultimately help to reduce the likelihood that they will recidivate. Further, this lack of treatment reflects poorly on these offenders, as participation in programming is generally considered when determining whether an offender should receive any form of early release (McGill, 2008). Despite the fact that programming is not available to many of these offenders, this is still counted against them, making it less likely that they will be released. This contributes directly to the overrepresentation of Aboriginal women in carceral institutions, as it makes them much more likely to serve the entirety of their sentence in prison.

\section{Culture and Correctional Programs}

While blocking female Aboriginal inmatesôaccess to programming is certainly problematic, the current state of programs in Canadian prisons should also be seen as a factor that contributes to the overrepresentation of Aboriginal women as offenders. As research indicates, many programs offered by the Correctional Service of Canada (CSC) are ñdesigned for male inmates and non-Aboriginal women, and then applied toé Aboriginal womenò(McGill, 2008, 101). This should be considered damaging to the well-being of female Aboriginal inmates, as they end up being treated in programs that are not only inappropriate for their gender, but for their cultural background as well. In many ways, this use of non-specific programming ignores the unique needs of these offenders, thereby reducing its efficacy. While this may be seen as a minor factor that is irrelevant to the programôs ability to function, research suggests that ñAboriginal people respond better to culturally appropriate rehabilitation services [than]..to mainstream rehabilitation servicesò (Milward, Parkes, 2011,89).

Although this only addresses the impact of culturally appropriate services, this finding can be extrapolated to other factors. If culturally appropriate services are more effective, it 
perhaps holds that the creation of gender-specific programming would be more effective as well. Additionally, while this research focuses on all Aboriginal offenders, it has also been suggested that the need for culturally specific programming is especially vital to the wellbeing of female Aboriginal inmates. This suggestion is based on the fact that this population is much more likely to be moved to facilities far away from cultural and familial support systems, due to a countrywide lack of womenôs facilities (McGill, 2008). As such, these women are much more isolated from their cultures than male Aboriginal inmates, which may have a damaging effect on their rehabilitation. This use of inappropriate rehabilitation programming has a major impact on the number of Aboriginal women in prison, as it is less likely to effectively treat the issues that contributed to their offending behavior.

Although specific and appropriate programming is vital to reducing the female Aboriginal population in prisons, an over-emphasis on culture can also be damaging to the rehabilitation of these offenders. In their present form, the programs offered to Aboriginal women treat them as a homogenous population, ultimately ñparticipat[ing] in the construction of an oversimplified, overgeneralized version of Aboriginal identityò (Martel \& Brassard, 2008, 344). This can have a negative impact on these offendersôrehabilitation in multiple ways. Primarily, these programs function on the basis that Aboriginal peoples want to be inundated with their own culture (Martel \& Brassard, 2008). This assumption is problematic, as it essentially forces Aboriginal offenders to participate in their culture, a practice which is not extended to offenders of any other ethnicity.

Again, as these programs are over-generalized, these offenders are often forced to adopt cultural practices and traditions that may not actually reflect their background. This creates a 
reluctance amongst this population to actually participate in these programs. While this contributes to the overpopulation of Aboriginal women in prison by failing to rehabilitate and discouraging participation in treatment programming, it can impact this overrepresentation in an even more direct manner. As research suggests, ñprisonersôparticipation in Aboriginal-centred programs is increasingly mandatory to their releaseò (Martel \& Brassard, 2008, 357). This should be considered a direct obstacle to the release of female Aboriginal inmates, as it forces them to choose between remaining in prison and participating in culturally specific programming that does not, in fact, reflect their culture. Ultimately, a balance must be reached between providing culturally appropriate, gender specific programming, and ensuring that this programming is not based on stereotypes and generalized notions about Aboriginal culture.

Additionally, while programming and treatment should be available, and participation should be encouraged, participating in programming that is culturally based should not be mandatory to an offenderôs release. Although treatment may be considered compulsory, the requirement that it be culturally specific places extra constraints on Aboriginal inmates, by forcing them to participate in a culture with which they may not wish to identify. Overall, the present state of programming is failing Aboriginal inmates, females in particular. The many flaws in the current system should be considered a direct cause of overrepresentation, both by failing to effectively rehabilitate these offenders and by keeping them incarcerated for longer periods of time if they fail to conform to the CSCô view of an appropriate expression of Aboriginal culture. 


\section{Conclusion}

Overall, Aboriginal women face a severe disadvantage at all levels of the justice system. In many ways, this disadvantage begins before the first contact with the justice system is even initiated, as the high levels of victimization that these women face as a result of their gender and cultural background places them at a much higher risk of offending than non-Aboriginal women. This disadvantage continues throughout their interaction with the justice system, whether it is through over-policing, discrimination at sentencing, or improper forms of classification and treatment in prisons. These factors work together to drastically increase the number of Aboriginal women in prison, and should be considered different stages in a cycle, as this systemic mistreatment further victimizes these women, and ultimately fails to provide both the consideration of their background, and the proper forms of rehabilitation that are required to help keep them out of prison. As a result, their contact with the justice system only serves to place them at a further disadvantage upon release, thus leaving them in a worse position than where they started. Despite this, the issue of overrepresentation of Aboriginal women in prisons cannot be considered solely the result of mistreatment by the justice system. Rather, this systemic discrimination should be seen as a reflection of the disadvantage that these women face in society at large. As such, a judicial reform should be undertaken to allow for proper consideration of the criminogenic factors that these women experience and to ultimately eliminate the discriminatory practices that occur at the hands of the justice system itself. 